\begin{tabular}{|c|c|}
\hline Title & Role of Wnt5a in suppressing invasiveness of hepatocellular carcinoma via epithelial-mesenchymal transition \\
\hline Author(s) & $\begin{array}{l}\text { Wakizaka, Kazuki; Kamiyama, Toshiya; W akayama, Kenji; Orimo, Tatsuya; Shimada, Shingo; Nagatsu, A kihisa; } \\
\text { Kamachi, Hirofumi; Y okoo, Hideki; Fukai, Moto; Kobay ashi, Nozomi; Mitsuhashi, T omoko; Taketomi, A kinobu }\end{array}$ \\
\hline Citation & $\begin{array}{l}\text { Oncology Letters, 20(5), } 268 \\
\text { https://doi.org/10.3892/ol.2020.12131 }\end{array}$ \\
\hline Issue Date & $2020-11$ \\
\hline Doc URL & http:/hdl.handle.net/2115/82493 \\
\hline Tyре & article \\
\hline Additional Information & There are other files related to this item in HUSCAP. Check the above URL. \\
\hline File Information & Oncol Lett 20 268.pdf \\
\hline
\end{tabular}

Instructions for use 


\title{
Role of Wnt5a in suppressing invasiveness of hepatocellular carcinoma via epithelial-mesenchymal transition
}

\author{
KAZUKI WAKIZAKA $^{1}$, TOSHIYA KAMIYAMA ${ }^{1}$, KENJI WAKAYAMA ${ }^{1}$, TATSUYA ORIMO ${ }^{1}$, \\ SHINGO SHIMADA ${ }^{1}$, AKIHISA NAGATSU ${ }^{1}$, HIROFUMI KAMACHI ${ }^{1}$, HIDEKI YOKOO ${ }^{1}$, \\ MOTO FUKAI $^{1}$, NOZOMI KOBAYASHI ${ }^{1}$, TOMOKO MITSUHASHI ${ }^{2}$ and AKINOBU TAKETOMI ${ }^{1}$ \\ ${ }^{1}$ Department of Gastroenterological Surgery I, Hokkaido University Graduate School of Medicine, \\ Sapporo, Hokkaido 060-8638; ${ }^{2}$ Department of Surgical Pathology, \\ Hokkaido University Hospital, Sapporo, Hokkaido 060-8648, Japan \\ Received January 18, 2020; Accepted July 14, 2020
}

DOI: $10.3892 /$ ol.2020.12131

\begin{abstract}
Inappropriate activation of the canonical Wnt signaling pathway is associated with progression of hepatocellular carcinoma (HCC). However, the association between the non-canonical pathway activated by Wnt5a and HCC is not well known. The present study investigated the significance of Wnt5a expression in HCC. Immunohistochemical staining of Wnt5a was performed on specimens from 243 patients who underwent hepatic resection for HCC. The present study investigated whether Wnt5a expression was associated with clinical and pathological factors and prognosis. Wnt5a expression in human HCC cell lines was investigated using western blotting. The effects of overexpression or knockdown of Wnt5a were evaluated using proliferation and invasion assays. Changes in epithelial-mesenchymal transition (EMT)-related molecules were investigated using western blotting. Wnt5a negativity was significantly associated with poor tumor differentiation and positive vascular invasion. In univariate analysis, Wnt5a negativity was identified as a significant prognostic factor for overall survival (OS). Multivariate analysis of OS demonstrated that Wnt5a negativity was an independent prognostic factor. Wnt5a expression was lower in HLE and HLF cells than in HepG2 and Huh7 cells. Knockdown of Wnt5a by short hairpin RNA transfection increased the proliferation and invasiveness of Huh7 cells, and decreased the expression levels of E-cadherin. In HLF cells, overexpression of Wnt5a inhibited invasiveness and decreased the expression levels of vimentin. Wnt5a negativity was associated with poor tumor differentiation and positive vascular invasion, and was an independent
\end{abstract}

Correspondence to: Dr Toshiya Kamiyama, Department of Gastroenterological Surgery I, Hokkaido University Graduate School of Medicine, N15 W7 South Research Building, Kita-ku, Sapporo, Hokkaido 060-8638, Japan

E-mail: t-kamiya@med.hokudai.ac.jp

Key words: Wnt5a, hepatocellular carcinoma, immunohistochemistry, epithelial-mesenchymal transition poor prognostic factor in patients with HCC. Wnt5a may be a tumor suppressor involved in EMT-mediated changes in invasiveness.

\section{Introduction}

Hepatocellular carcinoma (HCC) is the fifth most common cancer in the world (1). Although liver resection is one of the most effective treatments for $\mathrm{HCC}, \mathrm{HCC}$ has a high recurrence rate even after curative resection (2). To improve the prognosis of HCC, it is desirable to elucidate the mechanism of its carcinogenesis and progression and to identify target molecules for treatment.

The Wnt signaling pathway is classified into canonical and noncanonical pathways. Inappropriate activation of the former is associated with carcinogenesis via abnormal accumulation of cytoplasmic $\beta$-catenin and its translocation to the nucleus (3). However, the association between the noncanonical pathway, which does not involve activity of $\beta$-catenin, and carcinogenesis or tumor progression is not well known. In the Wnt signaling pathway, its ligands are the Wnt protein family, and there are 19 members in the family in mammals (4). Wnt5a is supposed to be the key ligand of the noncanonical pathway (5). The noncanonical pathway can be subclassified mainly into the planar cell polarity and $\mathrm{Ca}^{2+}$ pathways (6). Through these pathways, Wnt5a signaling plays an important role in regulating cell differentiation, proliferation, migration, adhesion and polarity (5). Abnormal activation or inhibition of Wnt5a signaling is associated with cancer progression or suppression (7). Several studies have shown that the function of Wnt5a differs depending on the type of cancer. For example, activation of Wnt5a has a suppressive effect on thyroid, colon and breast cancers (8-10), while it has a progressive effect on prostate, gastric and non-small cell lung cancers, and malignant melanoma (11-14). However, the effect of Wnt5a on HCC is not well known. Recent research has provided evidence that the WNT5A gene encodes two protein isoforms, termed Wnt5a-long (Wnt5a-L) and Wnt5a-short (Wnt5a-S). The two isoforms appear to have contrasting roles in cancer; that is, Wnt5a-L inhibits proliferation and Wnt5a-S increases proliferation in breast cancer, cervical cancer and neuroblastoma cell 
lines (15). In the present study, we investigated the significance of expression of Wnt5a in HCC.

\section{Materials and methods}

Patients and tissue samples. We retrospectively screened 243 patients (200 male and 43 female), with a median age of 63 years (range 35-82 years), who underwent hepatic resection for HCC between January 1997 and December 2006 at our institution. After surgery, patients were followed up by monitoring dynamic computed tomography and/or magnetic resonance imaging and tumor markers every 3 months on an outpatient basis. Combined examination of tumor markers and imaging studies was used for diagnosis of recurrence of HCC. We reviewed the medical records of all patients for clinical information, including sex, age, markers of hepatitis B virus and hepatitis $\mathrm{C}$ virus (HCV), serum albumin, serum a-fetoprotein (AFP), and protein induced by vitamin $\mathrm{K}$ absence or antagonist(PIVKA)II. We reviewed tumor size, tumor number, vascular invasion, lymph node metastasis, and the pathological findings of background liver. We used the criteria of the Liver Cancer Study Group of Japan (6th edition) to determine tumor-node-metastasis (TNM) stage of HCC (16). Informed consent of patients between 1997 and 2000 was obtained in the form of opt-out on the web site of Hokkaido University Hospital and patients between 2001 and 2006 signed the written informed consent. This research was approved by the Institutional Review Board of our institution (017-0237) and performed in compliance with the Declaration of Helsinki.

Immunohistochemical staining. Four-micrometer-thick sections of formalin-fixed and paraffin-embedded specimens were used for immunohistochemical staining. They were deparaffinized using xylene and ethanol, and antigen retrieval was performed using Target Retrieval Solution ( $\mathrm{pH} 9.0$; 415211; Nichirei Biosciences Inc., Tokyo, Japan), heated for $30 \mathrm{~min}$ at $95^{\circ} \mathrm{C}$. The samples were incubated with Block Ace (UKB80; $\mathrm{KAC}$ Co., Ltd.) for $5 \mathrm{~min}$ to block nonspecific antibody reactions and incubated overnight at $4^{\circ} \mathrm{C}$ with anti-Wnt5a antibody (LS-C47384, diluted 1:2,000; LifeSpan BioSciences Inc.). The samples were incubated in Histofine Simple Stain MAX PO (MULTI; 724152; Nichirei Biosciences) for $30 \mathrm{~min}$ at room temperature. Immunohistochemical staining was visualized using 3,3' diaminobenzidine, and sections were counterstained with hematoxylin. Immunoreactivity was evaluated according to the distribution of positive cells. Wnt5a-positive cells were defined according to the immunoreactivity on the cell membrane, regardless of cytoplasmic immunoreactivity. The immunohistochemical staining pattern of Wnt5a in HCC was heterogeneous and Wnt5a positivity was recorded if the proportion of Wnt5a-positive cells was $>50 \%$ (Fig. 1). Two authors who were blinded to the clinical and pathological parameters evaluated the results of immunohistochemical staining.

Cell lines. Human liver cancer cell lines HLE, HLF, HepG2 and Huh7 were obtained from the Japanese Collection of Research Bioresources Cell Bank (Osaka, Japan). They were authenticated by short tandem repeat profiling. They were cultured in Dulbecco's modified Eagle's medium containing
$10 \%$ fetal bovine serum (FBS). The medium was replaced every second day and all cell cultures were incubated at $37^{\circ} \mathrm{C}$ in $5 \% \mathrm{CO}_{2}$.

Cell transfection. To establish Wnt5a knockdown cells, we used a lentiviral short hairpin RNA (shRNA) targeting Wnt5a (TL320572; OriGene). Lenti-X HTX Packaging System (631251; Takara Bio Inc.) was used for transfection into cell lines. To create overexpressing cells, we obtained the full-length cDNA encoding human Wnt5a from cDNA of Huh7 cells by reverse transcription polymerase chain reaction. The Wnt5a primers were: Forward, 5'-CAGTGTGGTGGAATTGCC ACCATGAAGAAGTCC-3' and reverse, 5'-GATATCTGC AGAATTCTACTTGCACACAAACTGG-3'. The cDNA was cloned into pcDNA3.1 (V79020; Thermo Fisher Scientific, Tokyo, Japan). HLF cells were seeded in cell culture dishes, and after reaching $70 \%$ confluence, they were transfected with pcDNA3.1-Wnt5a overexpression vector. Lipofectamine 2000 (Invitrogen; Life Technologies) was used for cell transfection. The medium was replaced after $48 \mathrm{~h}$ with G418-containing medium, and stably transfected cells were selected using antibiotic resistance preferentially.

Western blotting. Cells were cultured to reach $80 \%$ confluence and harvested using lysis buffer on ice. A total of $10 \mu \mathrm{g}$ of each lysate was run on sodium dodecyl sulfate-polyacrylamide gel electrophoresis and transferred to polyvinylidene difluoride membranes. After blocking with $3 \%$ bovine serum albumin, membranes were immunoblotted using primary antibodies against Wnt5a (ab174963, diluted 1:500; Abcam); glyceraldehyde 3-phosphate dehydrogenase (GAPDH; \#3683, diluted 1:1,000; Cell Signaling Technology); E-cadherin (\#3195, diluted 1:1,000; Cell Signaling Technology); ZO-1 (\#8193, diluted 1:1,000; Cell Signaling Technology); N-cadherin (ab76011, diluted 1:5,000; Abcam); and vimentin (\#5741, diluted 1:1,000; Cell Signaling Technology). The blots were then reacted with secondary anti-rabbit antibodies (\#7074, diluted 1:5,000; Cell Signaling Technology), followed by detection of the proteins with SuperSignal West Dura Extended Duration Substrate (34076; Thermo Fisher Scientific).

Cell proliferation assay. Cells were seeded at 5,000/well in 96-well culture plates. We determined the number of viable cells by a colorimetric method using CellTiter 96 (G5430; Promega Corporation). The measurement was performed on 0 , 1,2 and 3 days after seeding.

Cell invasion assay. Cell invasion assays were performed in Matrigel invasion chambers ( 24 wells, $8 \mu \mathrm{m}$; 354480 ; Corning). Cells $\left(2.5 \times 10^{4}\right)$ in FBS-free medium were seeded in the upper chambers and medium containing 10\% FBS was added to the lower chambers. Cells above the membrane were wiped off using a cotton swab after $24 \mathrm{~h}$. Membranes were stained and average values were obtained by counting five fields per membrane under a microscope (x10).

DNA microarray. Purification of total RNA from HCC cell lines was performed using RNeasy Mini Kit (74106; Qiagen). Comprehensive analysis of gene expression was performed using SurePrint G3 Human 8x60 K version 3.0 (Agilent) at 

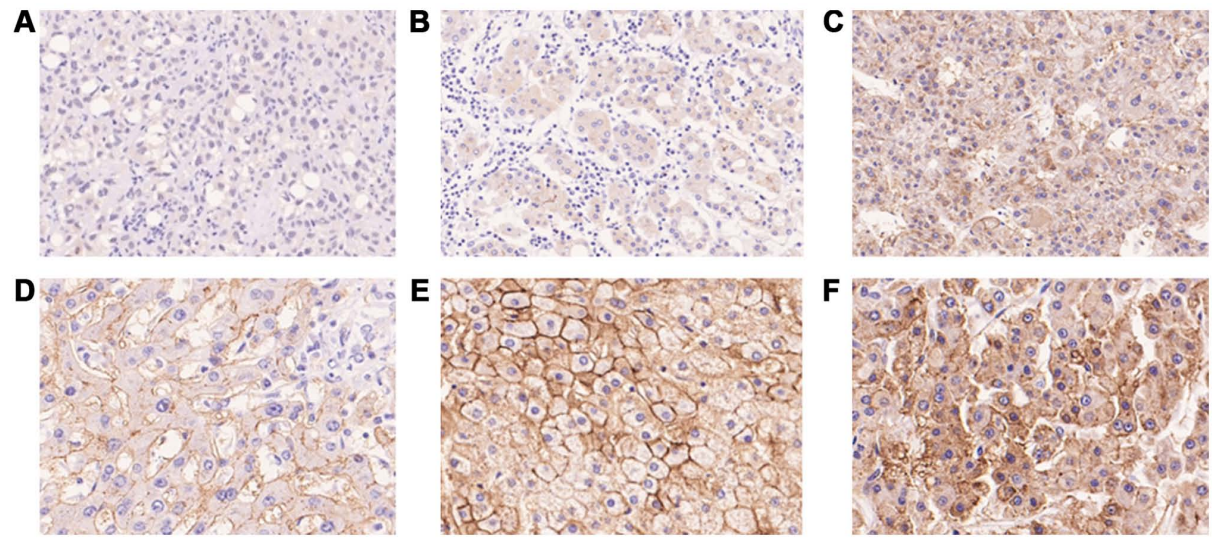

Figure 1. Representative images of immunohistochemical staining of Wnt5a in hepatocellular carcinoma tissues. Magnification, $\mathrm{x} 40$. The patterns of staining varied: (A) No staining, (B) weak cytoplasmic positive, (C) strong cytoplasmic positive, (D) weak cell membrane positive, (E) strong cell membrane positive, and (F) cytoplasmic and cell membrane both positive. (A-C) Positive staining only in the cytoplasm was classified as Wnt5a negative, and (D-F) cell membrane positive staining regardless of cytoplasmic staining was classified as Wnt5a positive.

Hokkaido System Science Co., Ltd. The fold changes in normalized signal values were used for comparison of gene expression levels.

Statistical analysis. Statistical analysis was performed using EZR version 1.35 (Saitama Medical Center, Jichi Medical University, Saitama, Japan) (17). Fisher's exact test was used to examine the correlation between expression of Wnt5a and clinical and pathological variables. The Kaplan-Meier method was used to plot OS and relapse-free survival (RFS) curves and they were compared using the log-rank test. The Cox proportional hazards regression model was used to perform multivariate analyses. Values in vitro were examined using Student's t-test. $\mathrm{P}<0.05$ was considered statistically significant.

\section{Results}

Clinical and pathological characteristics and Wnt5a expression in HCC patients. Clinical and pathological characteristics are summarized in Table I. Wnt5a expression was positive in 63 patients (25.9\%) and negative in 180 (74.1\%).

Correlations between clinical and pathological characteristics and Wnt5a expression are shown in Table II. Wnt5a negativity was significantly associated with $\mathrm{HCV}(\mathrm{P}=0.011)$, poor tumor differentiation $(\mathrm{P}=0.001)$ and positive vascular invasion $(\mathrm{P}=0.046)$.

Survival analysis. The 5-year OS rate in the Wnt5a-positive and Wnt5a-negative groups was 79.7 and 66.6\%, respectively. OS in the Wnt5a-negative group was significantly poorer than in the Wnt5a-positive group ( $\mathrm{P}=0.018)$. The 5-year $\mathrm{RFS}$ rate in the Wnt5a-positive and Wnt5a-negative groups was 43.7 and $30.7 \%$, respectively. RFS in the Wnt5a-negative group tended to be poorer than in the Wnt5a-positive group, but there was no significant difference between the groups (Fig. 2A and B).

Prognostic factor analysis. Univariate analysis showed that the following were predictive factors for lower OS in HCC patients: Albumin level [hazard ratio (HR) 1.826, 95\% confidence interval (CI) 1.204-2.770, P=0.005]; AFP level (HR 1.668, 95\% CI 1.090-2.553, P=0.018); PIVKAII level (HR 1.570,
95\% CI 1.002-2.459, $\mathrm{P}=0.049)$; tumor number $(\mathrm{HR} 1.824$, 95\% CI 1.134-2.932, $\mathrm{P}=0.013$ ); tumor size (HR 2.110, 95\% CI 1.384-3.218, $\mathrm{P}=0.001$ ); vascular invasion (HR 2.630, 95\% CI 1.645-4.205, $\mathrm{P}<0.001$ ); cirrhosis (HR 1.721, 95\% CI 1.130-2.623, $\mathrm{P}=0.012$ ); and Wnt5a negativity (HR 1.993, 95\% CI 1.109-3.368, $\mathrm{P}=0.020)$. Multivariate analysis showed that the following were predictive factors for lower OS: Albumin level (HR 1.571, 95\% CI 1.004-2.458, $\mathrm{P}=0.048$ ); tumor number (HR 1.965, 95\% CI 1.292-3.239, $\mathrm{P}=0.008$ ); tumor size (HR 1.829, 95\% CI 1.100-3.041, $\mathrm{P}=0.020)$; vascular invasion (HR 2.256, 95\% CI 1.317-3.865, $\mathrm{P}=0.003$ ); cirrhosis (HR 1.675, 95\% CI 1.075-2.610, $\mathrm{P}=0.023$ ); and Wnt5a negativity (HR 1.939, 95\% CI 1.076-3.497, P=0.028; Table III).

Analysis of recurrence. At the time of our investigation, 171 of the 243 patients $(70.4 \%)$ had experienced recurrence. This included 133 of the $180 \mathrm{Wnt5a-negative} \mathrm{patients}(73.9 \%)$ and 38 of the 63 Wnt5a-positive patients $(60.3 \%)$. The recurrence patterns and their management are summarized for each group (Table SI). The recurrence location did not differ significantly between the groups. There were also no significant differences in tumor number and diameter. The management strategies for recurrence varied, including transcatheter arterial chemoembolization, radiofrequency ablation, microwave coagulation therapy, percutaneous ethanol injection therapy, liver resection, metastatic resection, chemotherapy and radiotherapy. There was no significant difference in recurrence management between the Wnt5a-positive and Wnt5a-negative groups.

Prognosis after recurrence is shown in Fig. 2C and D. The 5-year OS after treatment of recurrence was $67.9 \%$ in the Wnt5a-positive group and $43.5 \%$ in the Wnt5a-negative group. OS after recurrence in the Wnt5a-negative group was significantly poorer than in the Wnt5a-positive group $(\mathrm{P}=0.026)$. Median RFS after treatment of recurrence was 10 months in the Wnt5a-positive group and 8 months in the Wnt5a-negative group. There was no significant difference in RFS after treatment of recurrence $(\mathrm{P}=0.576)$.

Wnt5a expression in liver cancer cell lines. Analysis using clinical samples revealed that Wnt5a expression was related 
Table I. Clinicopathological characteristics of patients.

\begin{tabular}{|c|c|}
\hline Characteristics & Value \\
\hline \multicolumn{2}{|l|}{ Sex, n $(\%)$} \\
\hline Male & $200(82.3)$ \\
\hline Female & $43(17.7)$ \\
\hline Median age, years (range) & $63(35-82)$ \\
\hline \multicolumn{2}{|l|}{ Viral infection, n (\%) } \\
\hline $\mathrm{HBV}$ & $90(37.0)$ \\
\hline $\mathrm{HCV}$ & $92(37.9)$ \\
\hline $\mathrm{HBV}+\mathrm{HCV}$ & $8(3.3)$ \\
\hline NBNC & $53(21.8)$ \\
\hline \multicolumn{2}{|l|}{ Child-Pugh class, n (\%) } \\
\hline A & $237(97.5)$ \\
\hline $\mathrm{B}$ & $6(2.5)$ \\
\hline \multicolumn{2}{|l|}{ Albumin, n (\%) } \\
\hline$<4 \mathrm{~g} / \mathrm{dl}$ & $96(39.5)$ \\
\hline$\geq 4 \mathrm{~g} / \mathrm{dl}$ & $147(60.5)$ \\
\hline \multicolumn{2}{|l|}{ AFP, n (\%) } \\
\hline$\leq 10 \mathrm{ng} / \mathrm{ml}$ & $127(52.3)$ \\
\hline$>10 \mathrm{ng} / \mathrm{ml}$ & $114(46.9)$ \\
\hline \multicolumn{2}{|l|}{ PIVKAII, n (\%) } \\
\hline$\leq 40 \mathrm{mAU} / \mathrm{ml}$ & $101(43.2)$ \\
\hline$>40 \mathrm{mAU} / \mathrm{ml}$ & $136(56.0)$ \\
\hline \multicolumn{2}{|l|}{ Differentiation, n (\%) } \\
\hline Well & $40(16.5)$ \\
\hline Moderate & $156(64.2)$ \\
\hline Poor & $47(19.3)$ \\
\hline \multicolumn{2}{|l|}{ Tumor number, n (\%) } \\
\hline Solitary & $187(77.0)$ \\
\hline Multiple & $56(23.0)$ \\
\hline \multicolumn{2}{|l|}{ Tumor size, n (\%) } \\
\hline$\leq 2 \mathrm{~cm}$ & $36(14.8)$ \\
\hline$>2-5 \mathrm{~cm}$ & $129(53.1)$ \\
\hline$>5-10 \mathrm{~cm}$ & $59(24.3)$ \\
\hline$>10 \mathrm{~cm}$ & $19(7.8)$ \\
\hline \multicolumn{2}{|l|}{ Vascular invasion, $\mathrm{n}(\%)$} \\
\hline Positive & $39(16.1)$ \\
\hline Negative & $204(83.9)$ \\
\hline \multicolumn{2}{|l|}{ Lymph node metastasis, $\mathrm{n}(\%)$} \\
\hline Positive & $0(0.0)$ \\
\hline Negative & $243(100.0)$ \\
\hline \multicolumn{2}{|l|}{ pStage $^{\mathrm{a}}, \mathrm{n}(\%)$} \\
\hline I & $24(9.9)$ \\
\hline II & $136(56.0)$ \\
\hline III & $83(34.2)$ \\
\hline IVA & $0(0.0)$ \\
\hline IVB & $0(0.0)$ \\
\hline \multicolumn{2}{|l|}{ Non-cancerous liver, n (\%) } \\
\hline Non-cirrhosis & $157(64.6)$ \\
\hline Cirrhosis & $86(35.4)$ \\
\hline \multicolumn{2}{|l|}{ Wnt5a, n (\%) } \\
\hline Positive & $63(25.9)$ \\
\hline Negative & $180(74.1)$ \\
\hline
\end{tabular}

a Liver Cancer Study Group of Japan, 6th Edition. Some categories did not include 243 patients due to incomplete data. HBV, hepatitis B virus; $\mathrm{HCV}$, hepatitis $\mathrm{C}$ virus; $\mathrm{NBNC}$, non-hepatitis $\mathrm{B}$ virus and non-hepatitis C virus; AFP, $\alpha$-fetoprotein; PIVKAII, protein induced by vitamin $\mathrm{K}$ absence or antagonist II.
Table II. Association between Wnt5a expression and clinicopathological characteristics.

\begin{tabular}{|c|c|c|c|}
\hline \multirow[b]{2}{*}{ Characteristics } & \multicolumn{2}{|c|}{ Wnt5a expression } & \multirow[b]{2}{*}{ P-value } \\
\hline & Negative, $\mathrm{n}$ & Positive, $\mathrm{n}$ & \\
\hline \multicolumn{4}{|l|}{ Sex } \\
\hline Male & 145 & 55 & 0.256 \\
\hline Female & 35 & 8 & \\
\hline \multicolumn{4}{|l|}{ Age, years } \\
\hline$\leq 60$ & 73 & 29 & 0.462 \\
\hline$>60$ & 107 & 34 & \\
\hline \multicolumn{4}{|l|}{$\mathrm{HBV}$} \\
\hline Negative & 110 & 35 & 0.459 \\
\hline Positive & 70 & 28 & \\
\hline \multicolumn{4}{|l|}{$\mathrm{HCV}$} \\
\hline Negative & 97 & 46 & $0.011^{\mathrm{a}}$ \\
\hline Positive & 83 & 17 & \\
\hline \multicolumn{4}{|l|}{ Albumin, g/dl } \\
\hline$<4$ & 68 & 28 & 0.372 \\
\hline$\geq 4$ & 112 & 35 & \\
\hline \multicolumn{4}{|l|}{ AFP, ng/ml } \\
\hline$\leq 10$ & 87 & 40 & 0.056 \\
\hline$>10$ & 91 & 23 & \\
\hline \multicolumn{4}{|c|}{ PIVKAII, mAU/ml } \\
\hline$\leq 40$ & 77 & 24 & 0.652 \\
\hline$>40$ & 99 & 37 & \\
\hline \multicolumn{4}{|l|}{ Differentiation } \\
\hline Well-moderate & 135 & 59 & $0.001^{\mathrm{a}}$ \\
\hline Poor & 45 & 4 & \\
\hline \multicolumn{4}{|l|}{ Tumor number } \\
\hline Solitary & 141 & 46 & 0.390 \\
\hline Multiple & 39 & 17 & \\
\hline \multicolumn{4}{|l|}{ Tumor size, $\mathrm{cm}$} \\
\hline$\leq 5$ & 125 & 40 & 0.434 \\
\hline$>5$ & 55 & 23 & \\
\hline \multicolumn{4}{|l|}{ Vascular invasion } \\
\hline Negative & 146 & 58 & $0.046^{\mathrm{a}}$ \\
\hline Positive & 34 & 5 & \\
\hline \multicolumn{4}{|c|}{ Non-cancerous liver } \\
\hline Non-cirrhosis & 118 & 39 & 0.647 \\
\hline Cirrhosis & 62 & 24 & \\
\hline
\end{tabular}

${ }^{\mathrm{a}} \mathrm{P}<0.05$. HBV, hepatitis $\mathrm{B}$ virus; $\mathrm{HCV}$, hepatitis $\mathrm{C}$ virus; $\mathrm{AFP}$, $\alpha$-fetoprotein; PIVKAII, protein induced by vitamin $\mathrm{K}$ absence or antagonist II.

to tumor differentiation, vascular invasion, and prognosis. Therefore, we used different cell lines to clarify the mechanism. We evaluated expression of Wnt5a in liver cancer cell lines using western blotting. We chose HLE and HLF as poorly differentiated cell lines, Huh7 as a well-differentiated cell line, and HepG2 as a hepatoma/liver cancer cell line. The intensity ratio of expression to GAPDH was $20.2 \%$ in HLE cells, $23.5 \%$ in HLF cells, $40.2 \%$ in HepG 2 cells and $85.5 \%$ in Huh7 cells 
A

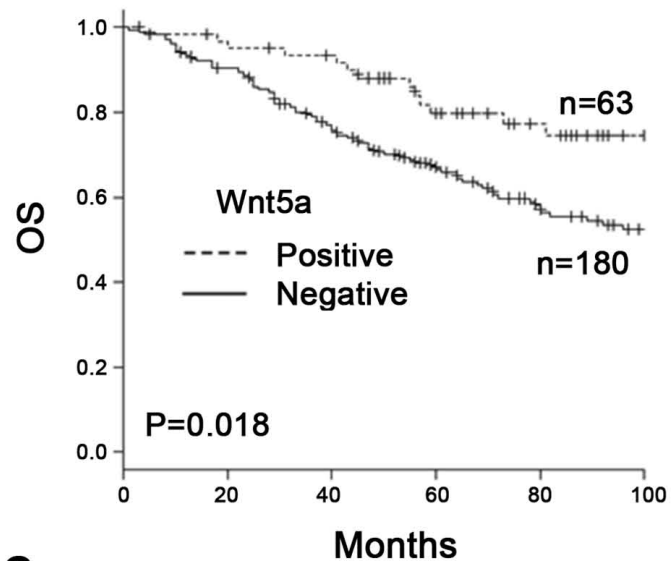

C

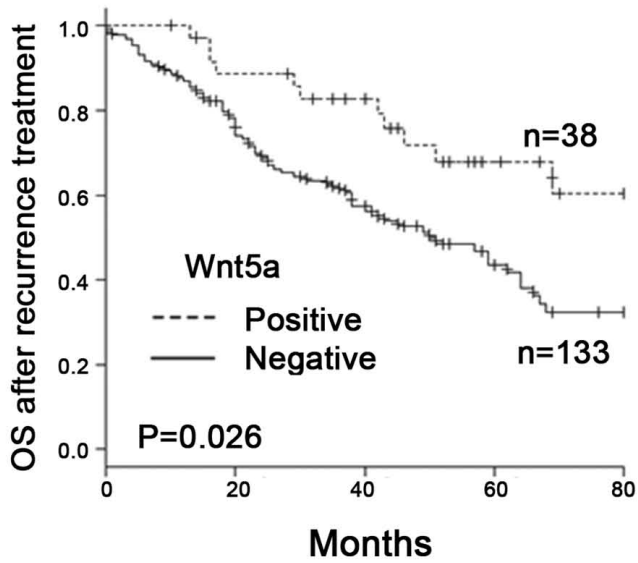

B
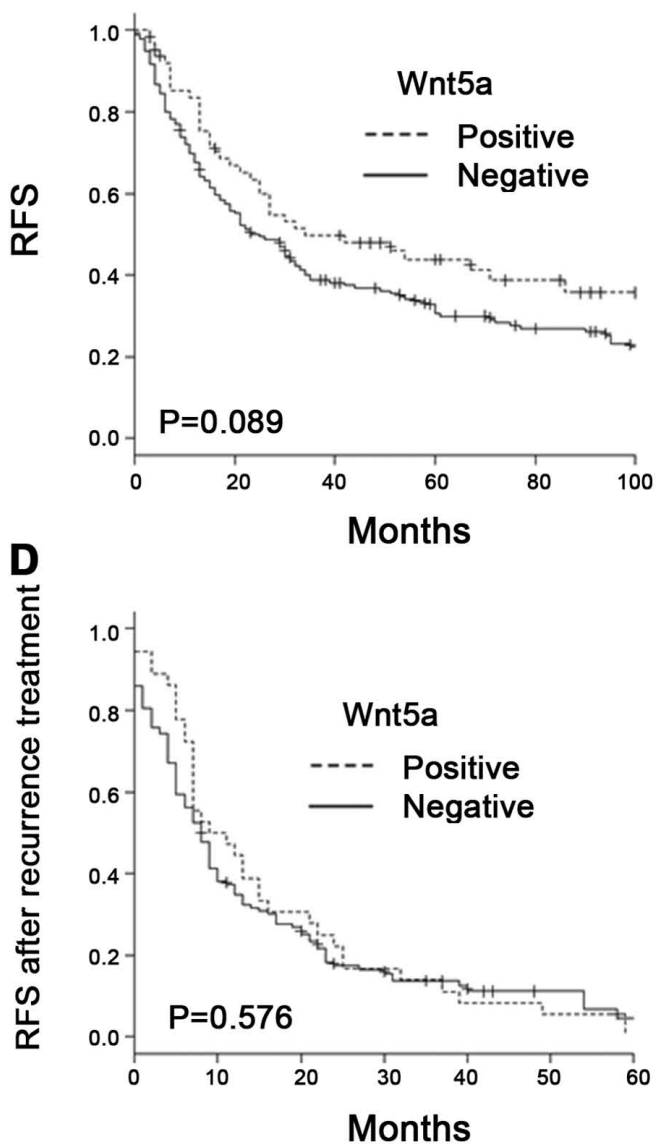

Figure 2. Survival analysis. (A) OS and (B) RFS curves were generated using the Kaplan-Meier method. OS in the Wnt5a-negative group was significantly lower than in the Wnt5a-positive group $(\mathrm{P}=0.018)$. RFS in the Wnt5a-negative group tended to be poorer than in the Wnt5a-positive group, but there was no significant difference ( $\mathrm{P}=0.089)$. (C) OS and (D) RFS curves after recurrence treatment were generated using the Kaplan-Meier method. OS after recurrence treatment in the Wnt5a-negative group was significantly poorer than in the Wnt5a-positive group $(\mathrm{P}=0.026)$. RFS after recurrence treatment showed no significant difference between the groups $(\mathrm{P}=0.576)$. OS, overall survival; RFS, relapse-free survival.

(Fig. S1). Wnt5a expression was lower in HLE and HLF cells than in HepG2 and Huh7 cells.

Effects of Wnt5a knockdown on Huh7 cells. Huh7 cells had high expression of Wnt5a, and we examined changes in proliferation and invasiveness of Huh7 cells by knockdown of Wnt5a. The cell proliferation assay showed that knockdown of Wnt5a significantly increased proliferation of Huh7 cells $(\mathrm{P}=0.043$; Fig. 3A). The invasion assay showed that knockdown of Wnt5a significantly increased invasiveness of Huh7 cells $(\mathrm{P}=0.009$; Fig. 3B).

Effects of Wnt5a overexpression on HLF cells. We examined changes in proliferation and invasiveness caused by overexpression of Wnt5a on HLF cells, which have low expression of Wnt5a. Overexpression of Wnt5a did not change proliferation of HLF cells (Fig. 3C) in the cell proliferation assay. Overexpression of Wnt5a significantly decreased invasiveness of HLF cells in the cell invasion assay ( $\mathrm{P}=0.003$; Fig. 3D).

Changes in EMT-related molecules by controlling Wnt5a expression. To elucidate the molecular mechanism involved in changes in invasiveness induced by Wnt5a, changes in EMT-related molecules were examined using western blotting (Fig. 4). We investigated changes in the expression of E-cadherin and ZO-1 as epithelial markers and $\mathrm{N}$-cadherin and vimentin as mesenchymal markers. Knockdown of Wnt5a in Huh7 cells with high expression of Wnt5a decreased E-cadherin expression, and there was no change in ZO-1, N-cadherin, or vimentin expression. Overexpression of Wnt5a in HLF cells with low expression of Wnt5a decreased vimentin expression, and there was no change in E-cadherin, ZO-1 or N-cadherin expression.

Changes in EMT-related genes by overexpression of Wnt $5 a$. To clarify the relationship between Wnt5a and EMT, we investigated changes in EMT-related genes with Wnt5a overexpression in HLF cells using DNA microarray analysis. The changes in SNAIL, ZEB and TWIST families as EMT transcriptional repressors are shown in Fig. S2. Expression of SNAI2 and SNAI3 was decreased by overexpression of Wnt5a. The changes in the matrix metalloproteinase (MMP) family are shown in Fig. S3. Expression of MMP2, MMP7, MMP9, MMP10 and MMP23B was decreased by overexpression of Wnt5a.

\section{Discussion}

In this study, Wnt5a negativity was associated with poor tumor differentiation and positive vascular invasion and 
Table III. Univariate and multivariate analysis of prognostic factors for overall survival.

\begin{tabular}{|c|c|c|c|c|c|c|}
\hline \multirow[b]{2}{*}{ Characteristics } & \multicolumn{3}{|c|}{ Univariate analysis } & \multicolumn{3}{|c|}{ Multivariate analysis } \\
\hline & HR & $95 \% \mathrm{CI}$ & P-value & $\mathrm{HR}$ & $95 \% \mathrm{CI}$ & P-value \\
\hline Sex (male vs. female) & 1.030 & $0.581-1.823$ & 0.921 & & & \\
\hline Age (>60 vs. $\leq 60$ years) & 1.426 & $0.926-2.196$ & 0.107 & & & \\
\hline HBV (positive vs. negative) & 0.873 & $0.569-1.339$ & 0.533 & & & \\
\hline $\mathrm{HCV}$ (positive vs. negative) & 1.132 & $0.743-1.724$ & 0.565 & & & \\
\hline Albumin (<4 vs. $\leq 4 \mathrm{~g} / \mathrm{dl})$ & 1.826 & $1.204-2.770$ & $0.005^{\mathrm{a}}$ & 1.571 & $1.004-2.458$ & $0.048^{\mathrm{a}}$ \\
\hline $\operatorname{AFP}(>10 \mathrm{vs} . \leq 10 \mathrm{ng} / \mathrm{ml})$ & 1.668 & $1.090-2.553$ & $0.018^{\mathrm{a}}$ & 1.483 & $0.953-2.307$ & 0.081 \\
\hline PIVKAII (>40 vs. $\leq 40 \mathrm{mAU} / \mathrm{ml}$ ) & 1.570 & $1.002-2.459$ & $0.049^{\mathrm{a}}$ & 1.468 & $0.883-2.439$ & 0.139 \\
\hline Tumor number (multiple vs. solitary) & 1.824 & $1.134-2.932$ & $0.013^{\mathrm{a}}$ & 1.965 & $1.292-3.239$ & $0.008^{\mathrm{a}}$ \\
\hline Tumor size ( $>5$ vs. $\leq 5 \mathrm{~cm})$ & 2.110 & $1.384-3.218$ & $0.001^{\mathrm{a}}$ & 1.829 & $1.100-3.041$ & $0.020^{\mathrm{a}}$ \\
\hline Vascular invasion (positive vs. negative) & 2.630 & $1.645-4.205$ & $<0.001^{\mathrm{a}}$ & 2.256 & $1.317-3.865$ & $0.003^{\mathrm{a}}$ \\
\hline Differentiation (poor vs. well + moderate) & 1.361 & $0.826-2.241$ & 0.226 & & & \\
\hline Non-cancerous liver (cirrhosis vs. non-cirrhosis) & 1.721 & $1.130-2.623$ & $0.012^{\mathrm{a}}$ & 1.675 & $1.075-2.610$ & $0.023^{\mathrm{a}}$ \\
\hline Wnt5a (negative vs. positive) & 1.933 & $1.109-3.368$ & $0.020^{\mathrm{a}}$ & 1.939 & $1.076-3.497$ & $0.028^{\mathrm{a}}$ \\
\hline
\end{tabular}

${ }^{a} \mathrm{P}<0.05$. HBV, hepatitis B virus; HCV, hepatitis C virus; NBNC, non-hepatitis B virus and non-hepatitis C virus; AFP, $\alpha$-fetoprotein; PIVKAII, protein induced by vitamin $\mathrm{K}$ absence or antagonist II; HR, hazard ratio.
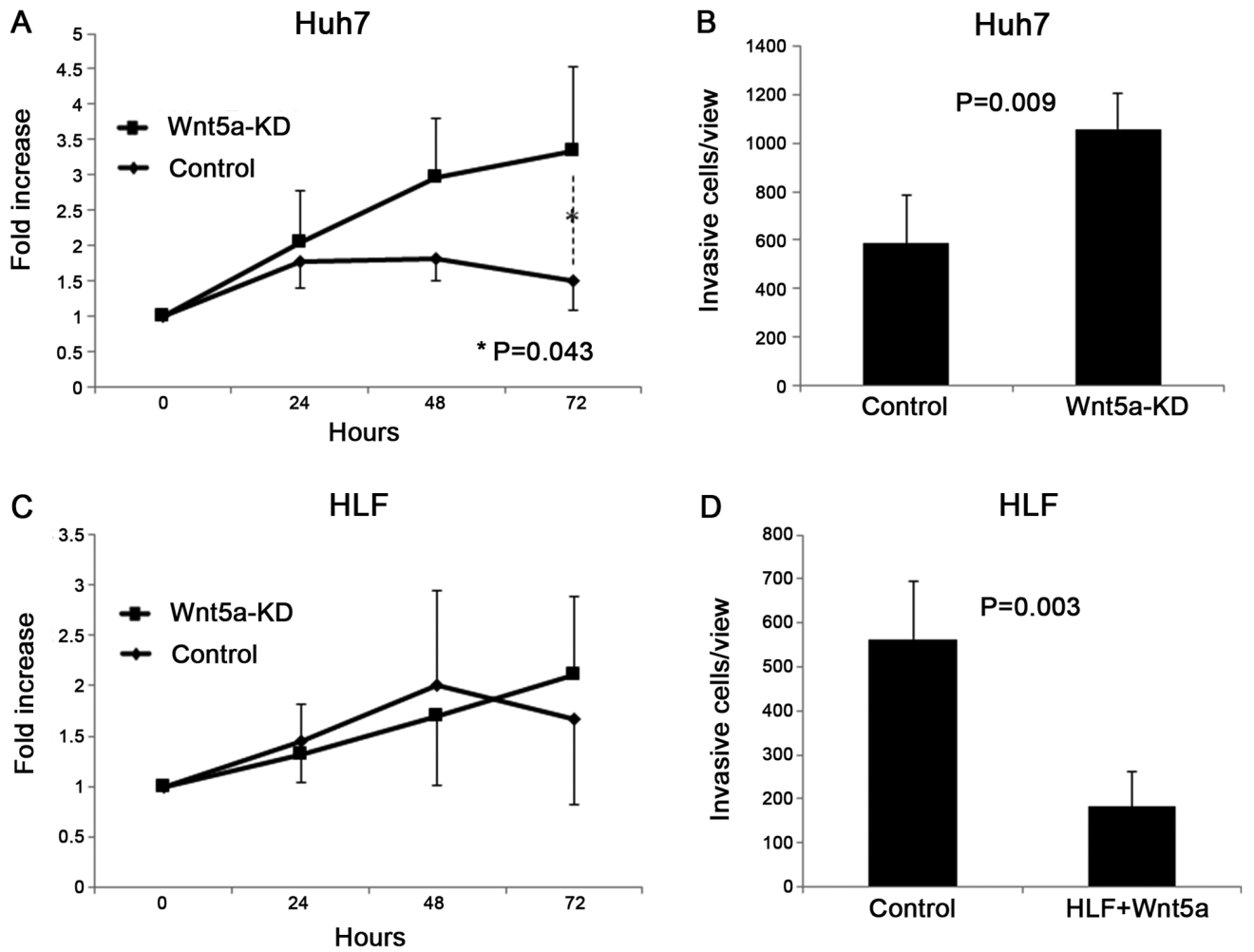

Figure 3. Effect of Wnt5a knockdown or overexpression on liver cancer cell lines. (A and B) Changes in proliferation and invasiveness of Huh7 cells following knockdown of Wnt5a. (A) Knockdown of Wnt5a significantly increased proliferation at $72 \mathrm{~h}$ after seeding of Huh7 cells ( $\mathrm{P}=0.04)$. (B) Knockdown of Wnt5a significantly increased invasiveness in Huh7 cells $(\mathrm{P}=0.009)$. (C and $\mathrm{D})$ Changes in proliferation and invasiveness of HLF cells following overexpression of Wnt5a. (C) Overexpression of Wnt5a did not change the proliferation of HLF cells. (D) Overexpression of Wnt5a significantly decreased the invasiveness of HLF cells $(\mathrm{P}=0.003)$. KD, knockdown.

was an independent poor prognostic factor in HCC patients. Knockdown of Wnt5a by shRNA increased the proliferation and invasiveness of Huh7 cells with high expression of Wnt5a, and decreased expression of E-cadherin. In HLF cells with low expression of Wnt5a, overexpression of Wnt5a inhibited invasiveness and decreased expression of vimentin. From the above, Wnt5a may be a tumor suppressor involved in EMT-mediated changes in invasiveness of HCC. Previous 

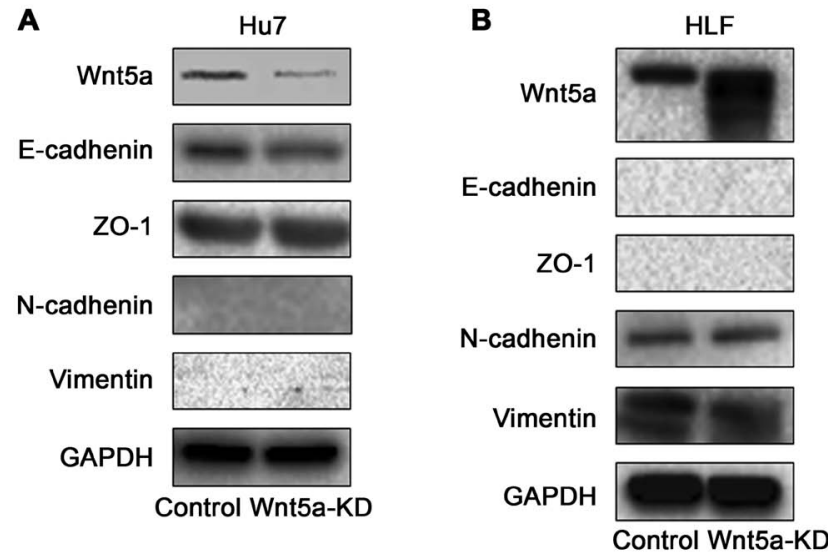

Figure 4. Changes in epithelial-mesenchymal transition-related molecules by controlling Wnt5a expression. The present study investigated the changes in the expression levels of E-cadherin and ZO-1 as epithelial markers and $\mathrm{N}$-cadherin and vimentin as mesenchymal markers. (A) Knockdown of Wnt5a in Huh7 cells with high expression levels of Wnt5a decreased E-cadherin expression, and there was no change in ZO-1, N-cadherin or vimentin expression. (B) Overexpression of Wnt5a in HLF cells with low expression levels of Wnt5a decreased vimentin expression, and there was no change in E-cadherin, ZO-1 or N-cadherin expression. KD, knockdown; ZO-1, zona occludens 1 .

studies have investigated the association of HCC and Wnt5a. They found that Wnt5a positivity is a good prognostic factor in HCC, and overexpression of Wnt5a in HCC cell lines suppresses cell proliferation $(9,18)$. These results may indicate that Wnt5a is a tumor suppressor in HCC. We validated these findings with a large sample in the present study.

Previous studies evaluated immunohistochemical staining of Wnt5a by cytoplasmic staining (19-21). We analyzed our data using the same methods as in previous studies (Tables SII and SIII, and Fig. S4). The results were almost the same as above except that Wnt5a was not significant in multivariate analysis. The appearance of Wnt5a immunostaining varied, and we focused on its expression on the cell membrane. Grouping by expression on the membrane made Wnt5a significant in multivariate analysis and it was considered to be a more dominant factor. It may be that expression of Wnt5a on the cell membrane means that Wnt5a is bound to its receptors and the non-canonical pathway is activated.

In this study, Wnt5a membrane negativity was correlated with poor pathological features such as poor differentiation, positive vascular invasion and poor prognosis. These results indicate that Wnt5a is a tumor suppressor in HCC. Previous studies supporting the tumor suppressor function of Wnt5a have shown that expression of Wnt5a is a good prognostic marker for long-term survival in colon cancer (22). Other research has shown that reduction of Wnt5a expression is correlated with increased serum AFP level and tumor stage, and that Wnt5a is an independent prognostic factor for $\operatorname{HCC}(18,23)$. The results of our investigation, with larger samples, support these previous results.

OS was significantly poorer in Wnt5a-negative than in Wnt5a-positive HCC patients. However, RFS was not significantly different between the groups. These results for OS and RFS were unexpected. To clarify the reason for this, the recurrence patterns and treatment were examined, and showed no difference between the two groups. HCC has a high frequency of recurrence caused by multicentric carcinogenesis (24), and this may cancel out the difference in recurrence. OS after recurrence in the Wnt5a-negative group was significantly poorer than in the Wnt5a-positive group despite receiving the same treatment for recurrence. This indicated that the responsiveness to treatment for recurrence was good because of the low malignancy of Wnt5a-positive tumors. We considered that expression of Wnt5a and malignancy of the tumor were related, and we used HCC cell lines to clarify the mechanism.

We investigated the expression of Wnt5a protein in liver cancer cell lines by western blotting. There are reports on the expression of Wnt5a in liver cancer cell lines at the mRNA level (25), but not at the protein level. In a previous study, Wnt5a was highly expressed in poorly differentiated cell lines at the mRNA level (25), but in our study, western blotting showed high expression of Wnt5a protein in well-differentiated cell lines. We also investigated mRNA expression (Fig. S5), but it was not correlated with protein expression. The high expression of Wnt5a protein in well-differentiated cell lines was consistent with the correlation between tumor differentiation and Wnt5a expression in our clinical study.

As a result of investigating the change in malignancy by controlling expression of Wnt5a, Wnt5a knockdown increased proliferation and invasiveness of Huh7 cells. Additionally, overexpression of Wnt5a did not change proliferation, but invasiveness was suppressed. Wnt5a is involved in the cytoskeleton and cell motility through the non-canonical Wnt pathway $(5,7)$. In our clinical investigation, there was a correlation between Wnt5a expression and vascular invasion, and it was thought that expression of Wnt5a was particularly related to changes in invasiveness. These results suggest that Wnt5a acts as a tumor suppressor through suppression of invasiveness of HCC cell lines.

In this study, we focused on EMT as a factor related to invasiveness. EMT is a process by which epithelial cells lose epithelial characteristics and acquire mesenchymal characteristics (26). EMT enables cell migration, which is a known mechanism of cancer invasion and metastasis (26). EMT epithelial markers include E-cadherin, ZO-1, claudins, occludin, cytokeratins and type IV collagen, and mesenchymal markers include N-cadherin, vimentin, FSP-1, a-SMA, fibronectin, and type I and III collagen (27). In our study, E-cadherin, ZO-1, N-cadherin and vimentin were used as representative markers. The association between Wnt5a and EMT has been reported in other cancer cell lines, but not for HCC $(22,28,29)$. Cheng et al (22) showed that overexpression of Wnt5a increased E-cadherin and decreased vimentin in colon cancer cell lines. ZEB1 and TWIST, which are repressors of E-cadherin, were decreased (22). Additionally, it is reported that SNAI1 and SNAI2 as transcriptional repressors promote EMT (30), and MMP family proteins such as MMP2 and MMP9 promote tumor cell invasion and metastasis through degradation of extracellular matrix in cooperation with EMT (31). In our study, knockdown of Wnt5a in Huh7 cells with high expression of Wnt5a decreased E-cadherin expression. Although overexpression of Wnt5a in HLF cells with low expression of Wnt5a did not increase E-cadherin, it decreased vimentin, SNAI2, MMP2 and MMP9, which are promoters of EMT. These changes in EMT-related factors indicate that Wnt5a is related to EMT and this is believed to 
be the first study to indicate a relationship between Wnt5a and EMT in HCC.

This study had some limitations. It was a retrospective single-center study. Further, prospective, multicenter studies are therefore necessary to validate our results. To clarify the relationship between Wnt5a and EMT in HCC, it is necessary to examine the signal transduction pathways involved. The Wnt5a isoforms Wnt5a-L and Wnt5a-S cannot be distinguished by the antibody and transfection method used in this study; therefore, it is not clear which one is significantly expressed or acts.

In conclusion, Wnt5a negativity was associated with poor tumor differentiation and positive vascular invasion, and it was an independent poor prognostic factor in HCC patients. Wnt5a may be a tumor suppressor involved in EMT-mediated changes of invasiveness. Expression of Wnt5a may be useful as a prognostic biomarker and affect the treatment after recurrence. Furthermore, this study may lead to the development of treatment for $\mathrm{HCC}$, focusing on the tumor-suppressing effect of Wnt5a.

\section{Acknowledgements}

The authors would like to thank Dr Cathel Kerr for editing a draft of this manuscript. This abstract was presented at the Gastrointestinal Cancer Symposium, January 23-25, 2020, San Francisco, CA, USA, and was published as Abstract no. 573.

\section{Funding}

The present study was supported by Japan Agency for Medical Research and Development (grant nos. JP19fk0310111 and JP19fk0210041), and in part by the Academic Support Program of Taiho Pharmaceutical Co., Ltd.

\section{Availability of data and materials}

The datasets used and/or analyzed during the current study are available from the corresponding author on reasonable request.

\section{Authors' contributions}

KWaki was involved in conceptualization, data curation, experiments and writing the original draft. TK designed the methodology, and was involved in supervision, and writing, review and editing. KWaka curated the data, performed experiments and supervised. TO, SS, AN and HK were involved in data curation and performed experiments. HY and MF curated data, performed experiments and supervised. NK was involved in data curation and performed experiments. TM designed the methodology and investigation. AT was involved in analysis and interpretation of data, funding acquisition, conceptualization, supervision, writing, review and editing. All authors read and approved the final manuscript.

\section{Ethics approval and consent to participate}

This research was approved by the Institutional Review Board of Hokkaido University Hospital (017-0237) and performed in compliance with the Helsinki Declaration. Informed consent of patients recruited between 1997 and 2000 was obtained in the form of opt-out on the web site of Hokkaido University Hospital and written informed consent was obtained from patients recruited between 2001 and 2006.

\section{Patient consent for publication}

Not applicable.

\section{Competing interests}

The authors declare that they have no competing interests.

\section{References}

1. Galle PR, Forner A, Llovet JM, Mazzaferro V, Piscaglia F, Raoul JL, Schirmacher P and Vilgrain V: EASL clinical practice guidelines: Management of hepatocellular carcinoma. J Hepatol 69: 182-236, 2018.

2. Kamiyama T, Nakanishi K, Yokoo H, Kamachi H, Tahara M, Suzuki T, Shimamura T, Furukawa H, Matsushita M and Todo S: Recurrence patterns after hepatectomy of hepatocellular carcinoma: Implication of Milan criteria utilization. Ann Surg Oncol 16: 1560-1571, 2009.

3. Nusse R and Clevers $H$ : Wnt/ $\beta$-catenin signaling, disease, and emerging therapeutic modalities. Cell 169: 985-999, 2017.

4. Logan CY and Nusse R: The Wnt signaling pathway in development and disease. Annu Rev Cell Dev Biol 20: 781-810, 2004.

5. Asem MS, Buechler S, Wates RB, Miller DL and Stack MS: Wnt5a signaling in cancer. Cancers (Basel) 8: 79, 2016.

6. van Amerongen R and Nusse R: Towards an integrated view of Wnt signaling in development. Development 136: 3205-3214, 2009.

7. Zhou Y, Kipps TJ and Zhang S: Wnt5a signaling in normal and cancer stem cells. Stem Cells Int 2017: 5295286, 2017.

8. Kremenevskaja N, Von Wasielewski R, Rao AS, Schöfl C, Andersson T and Brabant G: Wnt-5a has tumor suppressor activity in thyroid carcinoma. Oncogene 24: 2144-2154, 2005.

9. Dejmek J, Dejmek A, Säfholm A, Sjölander A and Andersson T: Wnt-5a protein expression in primary dukes B colon cancers identifies a subgroup of patients with good prognosis. Cancer Res 65 : 9142-9146, 2005.

10. Prasad CP, Chaurasiya SK, Guilmain W and Andersson T: WNT5A signaling impairs breast cancer cell migration and invasion via mechanisms independent of the epithelial-mesenchymal transition. J Exp Clin Cancer Res 35: 144, 2016.

11. Weeraratna AT, Jiang Y, Hostetter G, Rosenblatt K, Duray P Bittner $\mathrm{M}$ and Trent JM: Wnt5a signaling directly affects cell motility and invasion of metastatic melanoma. Cancer Cell 1: 279-288, 2002.

12. Wang B, Tang Z, Gong H, Zhu L and Liu X: Wnt5a promotes epithelial-to-mesenchymal transition and metastasis in non-small-cell lung cancer. Biosci Rep 37: BSR20171092, 2017.

13. Kanzawa M, Semba S, Hara S, Itoh T and Yokozaki H: WNT5A is a key regulator of the epithelial-mesenchymal transition and cancer stem cell properties in human gastric carcinoma cells. Pathobiology 80: 235-244, 2013.

14. Jin F, Qu X, Fan Q, Wang L, Tang T, Hao Y and Dai K: Regulation of prostate cancer cell migration toward bone marrow stromal cell-conditioned medium by Wnt5a signaling. Mol Med Rep 8: 1486-1492, 2013.

15. Bauer M, Bénard J, Gaasterland T, Willert K and Cappellen D: WNT5A encodes two isoforms with distinct functions in cancers. PLoS One 8: e80526, 2013.

16. The Liver Cancer Study Group of Japan: The General Rules for the Clinical and Pathological Study of Primary Liver Cancer. 6th edition. Kanehara \& Co., Ltd., Tokyo, 2015.

17. Kanda Y: Investigation of the freely available easy-to-use software 'EZR' for medical statistics. Bone Marrow Transplant 48: 452-458, 2013.

18. Geng M, Cao YC, Chen YJ, Jiang H, Bi LQ and Liu XH: Loss of Wnt5a and Ror2 protein in hepatocellular carcinoma associated with poor prognosis. World J Gastroenterol 18: 1328-1338, 2012 . 
19. Prgomet Z, Andersson T and Lindberg P: Higher expression of WNT5A protein in oral squamous cell carcinoma compared with dysplasia and oral mucosa with a normal appearance. Eur J Oral Sci 125: 237-246, 2017.

20. Zhong Z, Shan M, Wang J, Liu T, Shi Q and Pang D: Decreased Wnt5a expression is a poor prognostic factor in triple-negative breast cancer. Med Sci Monit 22: 1-7, 2016.

21. Kim Y, Hong M, Do IG, Ha SY, Lee D and Suh YL: Wnt5a, Ryk and Ror2 expression in glioblastoma subgroups. Pathol Res Pract 211: 963-972, 2015 .

22. Cheng R, Sun B, Liu Z, Zhao X, Qi L, Li Y and Gu Q: Wnt5a suppresses colon cancer by inhibiting cell proliferation and epithelial-mesenchymal transition. J Cell Physiol 229: 1908-1917, 2014.

23. Liu XH, Pan MH, Lu ZF, Wu B, Rao Q, Zhou ZY and Zhou XJ: Expression of Wnt-5a and its clinicopathological significance in hepatocellular carcinoma. Dig Liver Dis 40: 560-567, 2008.

24. Yang SL, Luo YY, Chen M, Zhou YP, Lu FR, Deng DF and Wu YR: A systematic review and meta-analysis comparing the prognosis of multicentric occurrence and vs intrahepatic metastasis in patients with recurrent hepatocellular carcinoma after hepatectomy. HPB (Oxford) 19: 835-842, 2017.

25. Yuzugullu H, Benhaj K, Ozturk N, Senturk S, Celik E, Toylu A, Tasdemir N, Yilmaz M, Erdal E, Akcali KC, et al: Canonical Wnt signaling is antagonized by noncanonical Wnt5a in hepatocellular carcinoma cells. Mol Cancer 8: 90, 2009.
26. Lamouille S, Xu J and Derynck R: Molecular mechanisms of epithelial-mesenchymal transition. Nat Rev Mol Cell Biol 15: 178-196, 2014.

27. Gonzalez DM and Medici D: Signaling mechanisms of the epithelial-mesenchymal transition. Sci Signal 7: re 8, 2014.

28. Xue Y, Zhang L, Zhu Y, Ke X, Wang Q and Min H: Regulation of proliferation and epithelial-to-mesenchymal transition (EMT) of gastric cancer by ZEB1 via modulating Wnt5a and related mechanisms. Med Sci Monit 25: 1663-1670, 2019.

29. Qin L, Yin YT, Zheng FJ, Peng LX, Yang CF, Bao YN, Liang YY, Li XJ, Xiang YQ, Sun R, et al: WNT5A promotes stemness characteristics in nasopharyngeal carcinoma cells leading to metastasis and tumorigenesis. Oncotarget 6: 10239-10252, 2015

30. Peinado H, Olmeda D and Cano A: Snail, Zeb and bHLH factors in tumour progression: An alliance against the epithelial phenotype? Nat Rev Cancer 7: 415-428, 2007.

31. Winer A, Adams S and Mignatti P: Matrix metalloproteinase inhibitors in cancer therapy: Turning past failures into future successes. Mol Cancer Ther 17: 1147-1155, 2018. 